\title{
Peptidyl-Prolyl Cis-Trans Isomerase A
}

National Cancer Institute

\section{Source}

National Cancer Institute. Peptidyl-Prolyl Cis-Trans Isomerase A. NCI Thesaurus. Code C156929.

Peptidyl-prolyl cis-trans isomerase A (165 aa, 18 kDa) is encoded by the human PPIA gene. This protein plays a role in cyclosporin-mediated immunosuppression, HIV virion assembly and protein folding. 